\title{
Preoperative Factors Affecting Postoperative Early Quality of Life During the Learning Curve of Holmium Laser Enucleation of the
} Prostate

\author{
Kang Jun Cho, Hyo Sin Kim, Jun Sung Koh, Seung Bum Han, Sang Hoon Kim, Hyun Woo Kim, Su Yeon Cho, Joon Chul Kim \\ Department of Urology, The Catholic University of Korea College of Medicine, Seoul, Korea
}

\begin{abstract}
Purpose: The aim of this study was to investigate the preoperative factors related to early quality of life (QoL) in patients with benign prostatic hyperplasia after holmium laser enucleation of the prostate (HoLEP) during the surgeon's learning curve.

Methods: The medical records of 82 patients with a follow-up period of at least 3 months who were treated with HoLEP during the time of a surgeon's learning curve were analyzed retrospectively. We divided the patients into two groups on the basis of the QoL component of the International Prostate Symptom Score (IPSS) 3 months after HoLEP: the high QoL group (IPSS/QoL $\leq 3$ ) and the low QoL group (IPSS/QoL $\geq 4$ ). Preoperative factors in each group were compared, including prostate volume, prostatespecific antigen, history of acute urinary retention (AUR), urgency incontinence, IPSS, and urodynamic parameters. Detrusor underactivity was defined as a bladder contractility index less than 100 on urodynamic study.

Results: A total of 61 patients (74.3\%) had a high QoL, whereas 21 (25.7\%) had a low QoL. A history of AUR, detrusor pressure on maximal flow (PdetQmax), bladder outlet obstruction grade, bladder contractility index, and detrusor underactivity were associated with postoperative QoL in the univariate analysis. In the multivariate analysis, a history of AUR and PdetQmax were independent factors affecting postoperative QoL.

Conclusions: A history of AUR and bladder contractility affect early QoL, and preoperative urodynamic study plays an important role in the proper selection of patients during the HoLEP learning curve.
\end{abstract}

Keywords: Prostatic hyperplasia; Holmium; Lasers; Quality of life

\section{INTRODUCTION}

Lower urinary tract symptoms associated with benign prostatic hyperplasia (BPH) are common and adversely affect quality of life (QoL) [1]. QoL decreases as urinary symptom severity increases but can be improved with treatment. The first-line treatment for symptomatic BPH is considered medical intervention, but there are several indications for surgery. Although transurethral resection of the prostate (TURP) is considered the gold standard for surgical treatment of $\mathrm{BPH}$, other new techniques for the surgical treatment of $\mathrm{BPH}$ have been introduced. Among the surgical techniques for $\mathrm{BPH}$, holmium laser enucleation of the prostate (HoLEP) has become an important treatment mo- dality, and several studies have shown that HoLEP is safe and effective for BPH of any size [2-5]. Improvement of the patient's QoL is one of the aims of HoLEP, and a significant improvement in QoL after HoLEP has been reported in some studies [6,7]. However, some patients show insufficient QoL improvement after HoLEP.

In addition, HoLEP is known to have a steep learning curve. Some reports have shown that an inexperienced urologist gains competence after 20 to 50 cases [8-10]. Thus, there are more unfavorable outcomes of HoLEP during the learning curve, and some members of the urologic community have been hesitant to embrace this technique. If we can predict unfavorable QoL outcomes before HoLEP and select patients accordingly during
Corresponding author: Joon Chul Kim

Department of Urology, The Catholic university of Korea, Bucheon St. Mary's Hospital, 327 Sosa-ro 327beon-gil, Wonmi-gu, Bucheon 420-717, Korea TEL: +82-32-340-7071 / FAX: +82-32-340-2124 / E-mail: kjc@catholic.ac.kr Submitted: June 10, 2013 / Accepted after revision: June 25, 2013
This is an Open Access article distributed under the terms of the Creative Commons Attribution Non-Commercial License (http://creativecommons.org/licenses/by-nc/3.0/) which permits unrestricted non-commercial use, distribution, and reproduction in any medium, provided the original work is properly cited. 
the learning curve, we can minimize these unfavorable outcomes and increase practitioner confidence in HoLEP. Despite numerous studies of HoLEP, however, few have sought to identify the factors predicting favorable and unfavorable QoL outcomes during the learning curve. In this study, we investigated the preoperative clinical factors and urodynamic parameters related to early QoL in patients with BPH after HoLEP during the surgeon's learning curve.

\section{MATERIALS AND METHODS}

We conducted a retrospective review of the clinical data of patients who underwent HoLEP for BPH performed by beginners. The patients included had undergone preoperative urodynamic study (UDS) and had been followed up for more than 3 months. All procedures were performed by one of six urologists who have considerable experience in endourological procedures. The procedures were performed without tutoring or a mentor with whom to discuss the procedure, and the learning process was self-directed and supplemented by watching edited videos of operations performed by experts and observing at another hospital. The first case of HoLEP was performed in December 2011, and the first 20 consecutive cases performed by each surgeon were enrolled. Transrectal needle biopsies of the prostate were performed to exclude prostate cancer when clinically necessary. Those who had prostate cancer diagnosed previously or after HoLEP, a history of prostatic or urethral surgery, disease with bladder outlet obstruction (BOO) other than $\mathrm{BPH}$, neurogenic bladder, or bladder cancer were excluded. Perioperative results, including enucleation efficiency (enucleation weight/enucleation time), morcellation efficiency (enucleation weight/morcellation time), and enucleation ratio (enucleation weight/transitional zone volume) were recorded.

The HoLEP procedure was begun by first dissecting the median and lateral prostate lobes off of the surgical capsule of the prostate in a retrograde direction from the apex and releasing them into the bladder. The equipment used consisted of a $100-\mathrm{W}$ holmium:yttrium-aluminum-garnet laser source (VersaPulse PowerSuite, Lumenis, Yokneam, Israel), a 550- $\mu$ m end-firing fiber (SlimLine, Lumenis), a modified continuous-flow 26-Fr resectoscope (Karl Storz, Tuttlingen, Germany), continuous saline solution irrigation, and a video system. The laser settings were $2.5 \mathrm{~J}$ and $40 \mathrm{~Hz}$. When enucleation was complete and hemostasis achieved, the enucleated tissue was evacuated from the bladder by use of a mechanical tissue morcellator (Versacut, Lumen- is) introduced through an indirect nephroscope (Karl Storz). At the end of the operation, a three-way 20-Fr Foley catheter was inserted, and the bladder was continuously irrigated. Usually, the patient was discharged after removal of the Foley catheter on the second day postoperatively.

The patients were divided into two groups with reference to the QoL component of their International Prostate Symptom Score (IPSS) 3 months after HoLEP: the high QoL group consisted of patients with an IPSS/QoL $\leq 3$, and the low QoL group consisted of patients with an IPSS/QoL $\geq 4$. Preoperative clinical factors were compared between the groups, including age, total prostate volume, transition zone volume, serum prostatespecific antigen (PSA) levels, history of acute urinary retention (AUR), urge incontinence, and IPSS. Prostate volumes were measured by use of transrectal ultrasonography. We also compared urodynamic parameters between groups, which included maximum flow rate (Qmax), post-void residual urine volume (PVR), maximum bladder capacity, detrusor pressure at the maximum flow rate (PdetQmax), BOO grade, BOO index, bladder contractility index (BCI), detrusor overactivity, and detrusor underactivity (DUA). BOO grade was measured by using the Schafer obstruction grade. The presence of detrusor overactivity was defined as either spontaneous or provoked involuntary detrusor contractions of $\geq 5 \mathrm{cmH}_{2} \mathrm{O}$ with urgency during filling cystometry. DUA was defined as a BCI less than 100 on UDS.

All statistical analyses were carried out by use of SAS ver. 9.2 (SAS Institute Inc., Cary, NC, USA). Continuous variables are reported as median (range) or mean \pm standard deviation and categorical variables are expressed as frequencies with percentages. The clinical and urodynamic characteristics were evaluated for statistically significant differences between the high QoL and low QoL groups by use of the Mann-Whitney U test for continuous variables and the chi-square and Fisher exact tests for categorical variables. We used logistic regression analysis to identify factors influencing QoL after HoLEP. Variables with Pvalues less than 0.05 in the univariate analysis were included in the multivariate logistic regression model (backward elimination). A $5 \%$ level of significance was used for all statistical testing.

\section{RESULTS}

Among 120 patients who were treated with HoLEP procedures, a total of 82 patients were enrolled in the study. The preoperative clinical and urodynamic characteristics of the patients are 
presented in Table 1. The two groups divided on the basis of their postoperative IPSS QoL score differed significantly in terms of history of AUR, PSA, PdetQmax, BOO grade, BCI, and DUA. The perioperative data of the enrolled patients are listed

Table 1. Baseline characteristics of enrolled patients

\begin{tabular}{|c|c|c|c|c|}
\hline \multirow{2}{*}{ Variable } & \multirow{2}{*}{ Total $(\mathrm{n}=82)$} & \multicolumn{2}{|c|}{ Post QoL } & \multirow{2}{*}{ P-value } \\
\hline & & $\operatorname{High}(0-3)(n=61)$ & Low $(4-6)(n=21)$ & \\
\hline Age (yr) & $69(52-85)$ & $68(52-85)$ & $72(59-84)$ & 0.120 \\
\hline History of AUR & $19(23.2)$ & $18(29.5)$ & $1(4.8)$ & $0.033^{\mathrm{a})}$ \\
\hline Urgency incontinence & $18(22.0)$ & $14(23.0)$ & $4(19.0)$ & 0.999 \\
\hline PSA (ng/mL) & $2.4(0.2-83.0)$ & $2.7(0.3-83.0)$ & $1.7(0.2-21.9)$ & $0.011^{\mathrm{a})}$ \\
\hline Total prostate volume $(\mathrm{mL})$ & $43(14-180)$ & $45.8(14-180)$ & $43(26.0-87.6)$ & 0.281 \\
\hline Transitional zone volume (mL) & $22.6(6-141)$ & $25(6-141)$ & $16(8.0-56.8)$ & 0.070 \\
\hline IPSS total & & & & 0.157 \\
\hline$<17$ & $32(41.6)$ & $26(46.4)$ & $6(28.6)$ & \\
\hline$\geq 17$ & $45(58.4)$ & $30(53.6)$ & $15(71.4)$ & \\
\hline IPSS storage symptom subscore & & & & 0.157 \\
\hline$<7$ & $32(41.6)$ & $26(46.4)$ & $6(28.6)$ & \\
\hline$\geq 7$ & $45(58.4)$ & $30(53.6)$ & $15(71.4)$ & \\
\hline IPSS voiding symptom subscore & & & & 0.113 \\
\hline$<12$ & $37(48.0)$ & $30(53.6)$ & $7(33.3)$ & \\
\hline$\geq 12$ & $40(52.0)$ & $26(46.4)$ & $14(66.7)$ & \\
\hline IPSS QoL score & & & & 0.275 \\
\hline $0-3$ & $22(28.2)$ & $18(31.6)$ & $4(19.0)$ & \\
\hline $4-6$ & $56(71.8)$ & $39(68.4)$ & $17(81.0)$ & \\
\hline \multicolumn{5}{|l|}{ Urodynamic parameters } \\
\hline $\mathrm{Qmax}(\mathrm{mL} / \mathrm{sec})$ & $9.1(1.6-21.0)$ & $9.0(1.6-21.0)$ & $9.3(2.4-18.8)$ & 0.447 \\
\hline Residual urine volume (mL) & $49(0-377)$ & $40(0-377)$ & $100(0-330)$ & 0.119 \\
\hline Maximum bladder capacity (mL) & $383(73-650)$ & $390(141-650)$ & $374(73-620)$ & 0.633 \\
\hline $\operatorname{PdetQmax}\left(\mathrm{cmH}_{2} \mathrm{O}\right)$ & $49(26-162)$ & $52(26-162)$ & $40(26-122)$ & $0.004^{\mathrm{a})}$ \\
\hline BOOI & & & & 0.075 \\
\hline$\leq 40$ & $49(59.8)$ & $33(54.1)$ & $16(76.2)$ & \\
\hline$>40$ & $33(40.2)$ & $28(45.9)$ & $5(23.8)$ & \\
\hline BOO grade & & & & $0.023^{\mathrm{a})}$ \\
\hline $3-6$ & $37(45.1)$ & $32(52.5)$ & $5(23.8)$ & \\
\hline $0-2$ & $45(54.9)$ & $29(47.5)$ & $16(76.2)$ & \\
\hline Bladder contractility index & $98.8(41-169)$ & $104.5(55-169)$ & $87(41-145.5)$ & $0.005^{\mathrm{a})}$ \\
\hline Detrusor overactivity & $15(18.3)$ & $11(18.0)$ & $4(19.0)$ & 0.999 \\
\hline Detrusor underactivity & $41(45.1)$ & $26(42.6)$ & $15(71.4)$ & $0.023^{\mathrm{a})}$ \\
\hline
\end{tabular}

Values are presented as median (range) or no. of patients (\%).

QoL, quality of life; AUR, acuter urinary retention; PSA, prostate specific antigen; IPSS, International Prostate Symptom Score; Qmax, maximal flow rate; PdetQmax, detrusor pressure on maximal flow; BOOI, bladder outlet obstruction index; BOO, bladder outlet obstruction.

a) Statistically significant. 
Table 2. Perioperative results of all patients $(n=82)$

\begin{tabular}{lc}
\hline Result & Mean \pm SD \\
\hline Enucleation time (min) & $90.8 \pm 42.5$ \\
Morcellation time (min) & $12.6 \pm 10.4$ \\
Used energy (KJ) & $144.9 \pm 78.4$ \\
Enucleation weight (g) & $17.9 \pm 14.0$ \\
Enucleation efficiency (g/min) & $0.19 \pm 0.12$ \\
Morcellation efficiency (g/min) & $1.98 \pm 1.71$ \\
Enucleation ratio & $0.59 \pm 0.30$ \\
Catheterization time (day) & $2.1 \pm 1.4$ \\
Hospital stay (day) & $3.9 \pm 1.2$ \\
\hline
\end{tabular}

$\mathrm{SD}$, standard deviation.

voiding symptom subscore $(\mathrm{P}<0.001)$, storage symptom subscore $(\mathrm{P}=0.001)$, and $\mathrm{QoL}$ score $(\mathrm{P}<0.001)$. Univariate analyses of preoperative factors affecting postoperative QoL revealed that a history of AUR, PdetQmax, BOO grade, BCI, and DUA were significant independent factors (Table 4). In the multivariate analysis, a history of AUR and PdetQmax were significantly associated with postoperative QoL (Table 5).

\section{DISCUSSION}

The present study examined early QoL after HoLEP performed by inexperienced surgeons during their learning curve. HoLEP has a longer learning curve than does standard TURP, and surgeons need at least 20 cases to achieve competence. The enucleation procedure is not easy, probably because of the difficulty in identifying the surgical plane for HoLEP and concern for complications such as stress urinary incontinence.

The overall enucleation efficiency $(0.19 \mathrm{~g} / \mathrm{min})$ in this study was relatively low. Ahyai et al. [11] recently reported an enucleation efficiency of $0.61 \mathrm{~g} / \mathrm{min}$ in small to medium prostates and $0.92 \mathrm{~g} / \mathrm{min}$ in large prostates during HoLEP performed by trained hands. However, that study also described the learning curve results, and the enucleation efficiency of a mean prostate volume of $53.4 \mathrm{~g}$ was $0.22 \mathrm{~g} / \mathrm{min}$ [12]. Despite the lower enucleation efficiency in our study, there were statistically significant improvements in voiding parameters 3 months postoperatively. Previous study groups also reported that clinical outcomes such as Qmax, PVR, and IPSS significantly improved postoperatively during the HoLEP learning curve $[9,13]$. However, $25.7 \%$ of patients in our study still had low QoL postoperatively, and their impaired QoL cannot be ignored. Our univariate analyses of
Table 3. Preoperative and postoperative voiding parameters $(\mathrm{n}=82)$

\begin{tabular}{lccl}
\hline Parameter & Preoperative & 1 Month & 3 Months \\
\hline Qmax $(\mathrm{mL} / \mathrm{sec})$ & $9.1 \pm 4.2$ & $14.5 \pm 7.1$ & $15.3 \pm 7.1$ \\
PVR $(\mathrm{mL})$ & $74.7 \pm 77.0$ & $21.8 \pm 19.9$ & $22.8 \pm 18.4$ \\
IPSS & & & \\
$\quad$ Total & $19.1 \pm 8.6$ & $12.7 \pm 7.6$ & $9.8 \pm 8.1$ \\
Storage symptom subscore & $7.7 \pm 3.8$ & $7.2 \pm 3.5$ & $5.6 \pm 4.0$ \\
Voiding symptom subscore & $11.5 \pm 5.8$ & $5.5 \pm 5.1$ & $4.1 \pm 4.9$ \\
QoL score & $4.2 \pm 1.0$ & $3.0 \pm 1.5$ & $2.3 \pm 1.5$ \\
\hline
\end{tabular}

Values are presented as mean \pm standard deviation.

Qmax, maximal flow rate; PVR, postvoid residual; IPSS, International Prostate Symptom Score; QoL, quality of life.

preoperative factors revealed that a history of AUR, high BOO grade, and preserved bladder contractility were associated with a high QoL during the HoLEP learning curve. A history of AUR and high PdetQmax were also associated with a high QoL in the multivariate analysis.

Patients with AUR secondary to BPH require prostate surgery. Although detrusor contractility could be reduced in chronic urinary retention, the safety and efficacy of HoLEP in patients with AUR has been proven. Elzayat et al. [14] demonstrated that the IPSS QoL score was significantly improved after HoLEP in patients with BPH and AUR. In the current study, the proportion of patients with a history of AUR was $29.5 \%$ in the high QoL group. This was higher than the $4.8 \%$ of patients with AUR in the low QoL group. A history of AUR was independently associated with postoperative QoL in the logistic regression analysis. The patients with AUR seemed to report a greater feeling of improvement in early QoL after relief of their LUTS by HoLEP. A comparison of patients with and without AUR treated with HoLEP indicated that those with AUR had a lower American Urologic Association Symptom Index and better QoL scores than did those without AUR, which suggests that patients with AUR might have a better outcomes secondary to the absence of preoperative storage symptoms [15]. Thus, surgeons should not hesitate to conduct HoLEP during the learning curve in patients with a history of AUR.

HoLEP can improve LUTS affecting QoL by enabling removal of the whole adenoma involved in urethral obstruction and release of the BOO. One of the reasons underlying the insufficient improvement of QoL with surgical treatment for BPH is that LUTS are caused not only by BOO but also by impaired detrusor contractility. Thus, preoperative urodynamic findings 
Table 4. Univariate analyses of preoperative factors affecting QoL after HoLEP

\begin{tabular}{|c|c|c|c|c|c|c|c|}
\hline Variable & $\begin{array}{c}\text { Odds } \\
\text { ratio }\end{array}$ & $95 \% \mathrm{CI}$ & P-value & Variable & $\begin{array}{c}\text { Odds } \\
\text { ratio }\end{array}$ & $95 \% \mathrm{CI}$ & P-value \\
\hline Age (yr) & 1.059 & $0.978-1.146$ & 0.156 & \multicolumn{4}{|l|}{ Urodynamic parameters } \\
\hline History of AUR & & & & $\mathrm{Qmax}(\mathrm{mL} / \mathrm{sec})$ & 1.055 & $0.939-1.186$ & 0.365 \\
\hline Presence & 1.000 & & & Residual urine volume (mL) & 1.005 & $0.999-1.011$ & 0.102 \\
\hline Absence & 8.371 & $1.043-67.160$ & 0.046 & Maximum bladder capacity & 0.999 & $0.995-1.003$ & 0.520 \\
\hline \multicolumn{4}{|l|}{ Urgency incontinence } & $(\mathrm{mL})$ & & & \\
\hline Absence & 1.000 & & & \multicolumn{4}{|l|}{ PdetQmax $\left(\mathrm{cmH}_{2} \mathrm{O}\right)$} \\
\hline Presence & 0.790 & $0.228-2.735$ & 0.710 & $\geq 50$ & 1.000 & & \\
\hline Prostate specific antigen & 0.886 & $0.747-1.050$ & 0.163 & $<50$ & 3.531 & $1.149-10.853$ & 0.028 \\
\hline Total prostate volume (mL) & & & & BOOI & & & \\
\hline$<40$ & 1.000 & & & $\leq 40$ & 1.000 & & \\
\hline$\geq 40$ & 1.128 & $0.396-3.214$ & 0.821 & $>40$ & 0.368 & $0.120-1.133$ & 0.081 \\
\hline Transitional zone volume (mL) & 0.968 & $0.929-1.009$ & 0.120 & \multicolumn{4}{|l|}{ BOO grade } \\
\hline IPSS total & & & & $3-6$ & 1.000 & & \\
\hline$<17$ & 1.000 & & & $0-2$ & 3.531 & $1.149-10.853$ & 0.028 \\
\hline$\geq 17$ & 2.167 & $0.734-6.397$ & 0.162 & Bladder contractility index & 0.970 & $0.949-0.991$ & 0.006 \\
\hline IPSS storage symptom subscore & & & & \multicolumn{4}{|l|}{ Detrusor overactivity } \\
\hline$<7$ & 1.000 & & & Absence & 1.000 & & \\
\hline$\geq 7$ & 2.167 & $0.734-6.397$ & 0.162 & Presence & 1.395 & $0.559-4.789$ & 0.753 \\
\hline IPSS voiding symptom subscore & & & & \multicolumn{4}{|l|}{ Detrusor underactivity } \\
\hline$<12$ & 1.000 & & & Absence & 1.000 & & \\
\hline$\geq 12$ & 2.308 & $0.809-6.583$ & 0.118 & Presence & 3.365 & $1.150-9.852$ & 0.027 \\
\hline \multicolumn{8}{|l|}{ IPSS QoL } \\
\hline $0-3$ & 1.000 & & & & & & \\
\hline $4-6$ & 1.962 & $0.577-6.671$ & 0.281 & & & & \\
\hline
\end{tabular}

QoL, quality of life; HoLEP, holmium laser enucleation of the prostate; CI, confidence interval; AUR, acute urinary retention; IPSS, International Prostate Symptom Score; Qmax, maximal flow rate; PdetQmax, detrusor pressure on maximal flow; BOOI, bladder outlet obstruction index; BOO, bladder outlet obstruction.

Table 5. Multivariate analyses of preoperative factors affecting QoL after HoLEP

\begin{tabular}{lccc}
\hline Variable & Odds ratio & 95\% CI & P-value \\
\hline $\begin{array}{l}\text { History of AUR } \\
\quad\end{array}$ & 1.000 & & \\
$\quad$ Presence & 9.253 & $1.122-76.319$ & 0.039 \\
$\quad$ Absence & & & \\
PdetQmax $\left(\mathrm{cmH}_{2} \mathrm{O}\right)$ & 1.000 & & \\
$\geq 50$ & 3.903 & $1.223-12.455$ & 0.021 \\
\hline 50 &
\end{tabular}

QoL, quality of life; HoLEP, holmium laser enucleation of the prostate; CI, confidence interval; AUR, acute urinary retention; PdetQmax, detrusor pressure on maximal flow. such as BOO and DUA affect the surgical outcome. Some previous studies have reported that the degree of BOO and DUA can affect the outcomes of TURP and laser vaporization of the prostate [16-18]. Those studies suggested that the efficacy of surgical treatment for $\mathrm{BPH}$ is significantly higher in patients with a worse preoperative degree of $\mathrm{BOO}$ and normal detrusor contractility. However, other studies have shown that the preoperative degree of BOO and DUA are weakly correlated with surgical outcomes [19-21].

However, most previous studies were based on the outcome of surgery for $\mathrm{BPH}$ performed by experts, and data for HoLEP are scarce in the literature. In the present study, the preoperative BOO grade in the high QoL group was significantly higher than 
that in the low QoL group. In addition, the low QoL group had preoperative urodynamic parameters indicative of weak detrusor contractility. Detrusor pressure is one of the parameters that determine the extent of $\mathrm{BOO}$ in the Schafer obstruction grade; thus, detrusor contractility in preoperative UDS affects postoperative early QoL during the HoLEP learning curve. Relieving the $\mathrm{BOO}$ by surgical treatment does not improve detrusor contractility [22]. Therefore, complete release of the $\mathrm{BOO}$ by HoLEP should be necessary to improve voiding symptoms and QoL in BPH patients with DUA. Complete relief of the obstruction with HoLEP may be difficult for a beginner. In this study, the mean enucleation ratio of the overall patient population was relatively low $(0.59 \pm 0.30)$. Bae et al. [10] reported an enucleation ratio of $0.66 \pm 0.35$ and a trend toward an improved enucleation ratio as a function of prostate volume. Although we did not analyze postoperative UDS nor the relationship between postoperative outcomes and enucleation ratio, the low enucleation ratio may imply that HoLEP was insufficient for relieving the obstruction.

This study had several limitations. First, we only used data from HoLEP performed during the learning curve and did not compare these data with outcomes of HoLEP performed by experienced hands. It is therefore unclear whether our findings were due to the fact that the procedure was performed by novices or were the result of flaws in the HoLEP procedure itself. However, our data could help with patient selection during the HoLEP learning curve. Second, during the learning curve, the rate of complications is known to be higher and to affect outcomes, but we did not specifically analyze data for complications. We focused on preoperative factors that affect the outcome of HoLEP, and there were no severe complications. HoLEP is known to provide equivalent outcomes in prostates of all sizes [23-25]. Prostate size did not affect postoperative QoL in this study. In general, beginners performing HoLEP tend to avoid treatment of large prostates because the degree of enucleation difficulty seems to depend on prostate size. The median total prostate volume in this study was $43.0 \mathrm{~mL}$, and this might be one limitation.

In conclusion, a history of AUR and preoperative bladder contractility affected early QoL during the HoLEP learning curve in this study. In general, history taking, physical examination, routine laboratory testing, and prostate ultrasound to assess prostate volume are performed in most $\mathrm{BPH}$ patients when surgical treatment is being considered. However, UDS in BPH has been performed for specific indications by some urologists.
BOO grade and bladder contractility can be measured by UDS. Thus, preoperative UDS plays an important role in the proper selection of patients during the HoLEP learning curve.

\section{CONFLICT OF INTEREST}

No potential conflict of interest relevant to this article was reported

\section{ACKNOWLEDGEMENTS}

The statistical consultation was supported by Catholic Research Coordinating Center of the Korea Health 21 R\&D Project (A070001), Ministry of Health \& Welfare, Republic of Korea.

\section{REFERENCES}

1. Trueman P, Hood SC, Nayak US, Mrazek MF. Prevalence of lower urinary tract symptoms and self-reported diagnosed 'benign prostatic hyperplasia, and their effect on quality of life in a communitybased survey of men in the UK. BJU Int 1999;83:410-5.

2. Krambeck AE, Handa SE, Lingeman JE. Experience with more than 1,000 holmium laser prostate enucleations for benign prostatic hyperplasia. J Urol 2013;189(1 Suppl):S141-5.

3. Gilling PJ, Aho TF, Frampton CM, King CJ, Fraundorfer MR. Holmium laser enucleation of the prostate: results at 6 years. Eur Urol 2008;53:744-9.

4. Kuntz RM, Lehrich K, Ahyai SA. Holmium laser enucleation of the prostate versus open prostatectomy for prostates greater than 100 grams: 5-year follow-up results of a randomised clinical trial. Eur Urol 2008;53:160-6.

5. Bae J, Choo M, Park JH, Oh JK, Paick JS, Oh SJ. Holmium laser enucleation of prostate for benign prostatic hyperplasia: seoul national university hospital experience. Int Neurourol J 2011;15:29-34.

6. Vavassori I, Valenti S, Naspro R, Vismara A, Dell'Acqua V, Manzetti A, et al. Three-year outcome following holmium laser enucleation of the prostate combined with mechanical morcellation in 330 consecutive patients. Eur Urol 2008;53:599-604.

7. Elmansy HM, Kotb A, Elhilali MM. Holmium laser enucleation of the prostate: long-term durability of clinical outcomes and complication rates during 10 years of followup. J Urol 2011;186:1972-6.

8. El-Hakim A, Elhilali MM. Holmium laser enucleation of the prostate can be taught: the first learning experience. BJU Int 2002;90: 863-9.

9. Shah HN, Mahajan AP, Sodha HS, Hegde S, Mohile PD, Bansal MB. 
Prospective evaluation of the learning curve for holmium laser enucleation of the prostate. J Urol 2007;177:1468-74.

10. Bae J, Oh SJ, Paick JS. The learning curve for holmium laser enucleation of the prostate: a single-center experience. Korean J Urol 2010; 51:688-93.

11. Ahyai SA, Chun FK, Lehrich K, Dahlem R, Zacharias MS, Fisch MM, et al. Transurethral holmium laser enucleation versus transurethral resection of the prostate and simple open prostatectomy: which procedure is faster? J Urol 2012;187:1608-13.

12. Elzayat EA, Elhilali MM. Holmium laser enucleation of the prostate (HoLEP): long-term results, reoperation rate, and possible impact of the learning curve. Eur Urol 2007;52:1465-71.

13. Jeong CW, Oh JK, Cho MC, Bae JB, Oh SJ. Enucleation ratio efficacy might be a better predictor to assess learning curve of holmium laser enucleation of the prostate. Int Braz J Urol 2012;38:362-71.

14. Elzayat EA, Habib EI, Elhilali MM. Holmium laser enucleation of prostate for patients in urinary retention. Urology 2005;66:789-93.

15. Anderson CB, Helfand BT, McVary KT. Holmium laser prostatic resection for patients presenting with acute urinary retention. BJU Int 2008;102:1623-8.

16. Tanaka Y, Masumori N, Itoh N, Furuya S, Ogura H, Tsukamoto T. Is the short-term outcome of transurethral resection of the prostate affected by preoperative degree of bladder outlet obstruction, status of detrusor contractility or detrusor overactivity? Int J Urol 2006;13: 1398-404.

17. Javle P, Jenkins SA, Machin DG, Parsons KF. Grading of benign prostatic obstruction can predict the outcome of transurethral prostatectomy. J Urol 1998;160:1713-7.
18. Paick JS, Um JM, Kwak C, Kim SW, Ku JH. Influence of bladder contractility on short-term outcomes of high-power potassium-titanyl-phosphate photoselective vaporization of the prostate. Urology 2007;69:859-63.

19. Oh MM, Kim JW, Kim JJ, Moon du G. Is there a correlation between the outcome of transurethral resection of prostate and preoperative degree of bladder outlet obstruction? Asian J Androl 2012;14:556-9.

20. Han DH, Jeong YS, Choo MS, Lee KS. The efficacy of transurethral resection of the prostate in the patients with weak bladder contractility index. Urology 2008;71:657-61.

21. Seki N, Yunoki T, Tomoda T, Takei M, Yamaguchi A, Naito S. Association among the symptoms, quality of life and urodynamic parameters in patients with improved lower urinary tract symptoms following a transurethral resection of the prostate. Neurourol Urodyn 2008;27:222-5.

22. Al-Hayek S, Thomas A, Abrams P. Natural history of detrusor contractility: minimum ten-year urodynamic follow-up in men with bladder outlet obstruction and those with detrusor. Scand J Urol Nephrol Suppl 2004;(215):101-8.

23. Humphreys MR, Miller NL, Handa SE, Terry C, Munch LC, Lingeman JE. Holmium laser enucleation of the prostate: outcomes independent of prostate size? J Urol 2008;180:2431-5.

24. Shah HN, Sodha HS, Kharodawala SJ, Khandkar AA, Hegde SS, Bansal MB. Influence of prostate size on the outcome of holmium laser enucleation of the prostate. BJU Int 2008;101:1536-41.

25. Seki N, Tatsugami K, Naito S. Holmium laser enucleation of the prostate: comparison of outcomes according to prostate size in 97 Japanese patients. J Endourol 2007;21:192-6. 